\title{
Large deviations for the Bessel clock
}

\author{
MARC YOR ${ }^{1}$ and MARGUERITE ZANI ${ }^{2}$ \\ ${ }^{1}$ Laboratoire de Probabilités et Modèles Aléatoires, Université Paris VI, 4 place Jussieu, \\ Tour 56, 75252 Paris, Cedex 5, France \\ ${ }^{2}$ Laboratoire de Mathématiques, Bât. 425, Université Paris Sud, 91405 Orsay Cedex, France. \\ E-mail: marguerite.zani@math.u-psud.fr
}

We show the law of large numbers, the central limit theorem and the large-deviation principle for the Bessel clock $\int_{0}^{t} \mathrm{~d} s /\left(R_{s}^{(v)}\right)^{2}$, where $\left(R_{t}^{(v)}, t \geqslant 0\right)$ is a Bessel process of index $v>0$. We also give functional versions of these limit theorems.

Keywords: Bessel processes; Brownian motion; large deviations

\section{Introduction}

Consider $\left(B_{t}, t \geqslant 0\right)$, a Brownian motion (BM) in $\mathbb{R}^{d}$, with $d \geqslant 2$, and $B_{0} \neq 0$ almost surely (a.s.). It is well known that $\left(B_{t}, t \geqslant 0\right)$ a.s. does not visit 0 , hence the increasing process

$$
C_{t}=\int_{0}^{t} \frac{\mathrm{d} s}{\left|B_{s}\right|^{2}}, \quad t \geqslant 0,
$$

is finite. We shall call this process the Bessel clock, since, on the one hand, it involves only the Bessel process $\left(\left|B_{t}\right|, t \geqslant 0\right)$ and, on the other hand, $\left(C_{t}, t \geqslant 0\right)$ is the time-change, or 'clock', which appears in the skew product decomposition

$$
B_{t}=\left|B_{t}\right| \theta_{C_{t}}
$$

of $\left(B_{t}, t \geqslant 0\right)$ into its radial part $\left(\left|B_{t}\right|, t \geqslant 0\right)$ and its angular part $B_{t} /\left|B_{t}\right|=\theta_{C_{t}}$, where $\left(\theta_{u}, u \geqslant 0\right)$ is a $\mathrm{BM}$ taking values in $S_{d-1}$, the unit sphere of $\mathbb{R}^{d}$, and independent of $\left(\left|B_{t}\right|, t \geqslant 0\right)$ (see, for example, Itô and McKean 1974; Revuz and Yor 1999).

More generally, if $\left(R_{t}^{(1)}, t \geqslant 0\right)$ and $\left(R_{t}^{(2)}, t \geqslant 0\right)$ are two independent Bessel processes with respective dimensions $d_{1}$ and $d_{2}$, and if $d_{1}+d_{2} \geqslant 2$, and $R_{0}^{(1)}+R_{0}^{(2)}>0$ a.s., then there is the skew product representation

$$
R_{t}^{(1)}=\rho_{t} Y_{C_{t}^{(v)}}
$$

where

$$
C_{t}^{(v)}=\int_{0}^{t} \frac{\mathrm{d} s}{\rho_{s}^{2}}
$$

and $v=\left(d_{1}+d_{2}-2\right) / 2$, and where $\rho_{t}=\left\{\left(R_{t}^{(1)}\right)^{2}+\left(R_{t}^{(2)}\right)^{2}\right\}^{1 / 2}$ is a Bessel process with dimension $d_{1}+d_{2}$, independent of $\left(Y_{u}, u \geqslant 0\right)$, a so-called Jacobi process with dimensions $\left(d_{1}, d_{2}\right)$. See, for example, Warren and Yor $(1997 ; 1998)$ for a discussion of these processes, 
and $\mathrm{Hu}$ et al. (1999) for some applications to asymptotics of diffusions in (Brownian) random media. See also Karlin and Taylor (1981).

A third natural occurrence of the Bessel clock concerns geometric Brownian motion $\left(\exp \left(\beta_{t}+v t\right) ; t \geqslant 0\right)$ which may be represented in Lamperti's form as

$$
\exp \left(\beta_{t}+v t\right)=R_{A_{t}^{(v)}}^{(v)}
$$

where $\left(R_{u}^{(v)}, u \geqslant 0\right)$ is a Bessel process with dimension $d=2(1+v)$ and

$$
A_{t}^{(v)}=\int_{0}^{t} \mathrm{~d} s \exp 2\left(\beta_{s}+v s\right) .
$$

Here, of course, $R^{(v)}$ and $A^{(v)}$ are not at all independent. In fact, they are closely related and, in particular,

$$
\left(A_{t}^{(v)}>u\right)=\left(t>C_{u}^{(v)} \equiv \int_{0}^{u} \frac{\mathrm{d} s}{\left(R_{s}^{(v)}\right)^{2}}\right),
$$

that is to say, the inverse of $A^{(v)}$ is the Bessel clock associated with $R^{(v)}$. This relationship and the knowledge of the law of $C^{(v)}$ obviously yield some results on $A_{t}^{(v)}$; see, for example, Yor (1992) and Geman and Yor (1993) for some applications to the pricing of Asian options.

The previous discussion shows that there is some interest in studying the asymptotic behaviour of $\left(C_{t}^{(v)} ; t \rightarrow+\infty\right)$, the Bessel clock associated with a Bessel process of dimension $d>2$. The following law of large numbers (LLN) and central limit theorem (CLT) are essentially given in Revuz and Yor (1999).

Theorem 1.1. Let $\left(R_{t}^{(v)}, t \geqslant 0\right)$ be a Bessel process starting from $R_{0}^{(v)} \neq 0$ a.s., with dimension $d>2$, i.e. $v>0$. Then we have

$$
\begin{aligned}
& \frac{1}{\log t} C_{t}^{(v)} \underset{t \rightarrow+\infty}{\longrightarrow} \frac{1}{d-2}=\frac{1}{2 v} \text { a.s. and in } L^{p} ; \\
& \sqrt{\log t}\left(\frac{1}{\log t} \int_{0}^{t} \frac{\mathrm{d} s}{\left(R_{s}^{(v)}\right)^{2}}-\frac{1}{d-2}\right) \underset{t \rightarrow+\infty}{\stackrel{\text { law }}{\longrightarrow}} N,
\end{aligned}
$$

where $N$ is centred Gaussian with variance $\sigma^{2}=1 / 2 v^{3}$.

Proof. These results are stated respectively as Exercise (4.23), Chapter IV and Exercise (3.20), Chapter $\mathrm{X}$ in Revuz and Yor (1999). In fact, in these exercises $\left(R_{t}, t \geqslant 0\right)$ denotes the radial part of the $\mathrm{BM}$ in $\mathbb{R}^{d}, d>2$, but the proofs easily extend to Bessel processes (as defined and studied in Revuz and Yor 1999, Chapter XI) with dimension $d>2$. To give a few details, we show how (8) follows from (7). In this proof, we skip the exponent $v$ for the sake of clarity. From Itô's formula,

$$
\log R_{t}=\log R_{0}+\int_{0}^{t} \frac{\mathrm{d} \beta_{s}}{R_{s}}+\frac{d-2}{2} \int_{0}^{t} \frac{\mathrm{d} s}{R_{s}^{2}}
$$

hence 


$$
\sqrt{\log t}\left(\frac{d-2}{2 \log t} \int_{0}^{t} \frac{\mathrm{d} s}{R_{s}^{2}}-\frac{1}{2}\right)
$$

has the same asymptotic law as

$$
\frac{1}{\sqrt{\log t}} \int_{0}^{t} \frac{\mathrm{d} \beta_{s}}{R_{s}}=\gamma_{s_{t}}
$$

where

$$
S_{t}=\frac{C_{t}^{(v)}}{\log t}
$$

and where $\left(\gamma_{u}, u \geqslant 0\right)$ is a (Dubins-Schwarz) BM obtained after 'log-scaling'. Finally, from (7) the right-hand term of equality (10) converges in law towards

$$
\gamma_{1 / 2 v} \stackrel{\text { law }}{=} \frac{1}{\sqrt{2 v}} \gamma_{1} \text {. }
$$

Since Bessel processes form the core of this paper, we devote the following section to their most important properties. The remainder of the paper is organized as follows. In Section 3, we complement the result (7) in Theorem 1.1 with a large-deviation principle (LDP). In Section 4, we give functional versions of the LLN, CLT and LDP for $\left\{C_{t}^{(v)}\right\}$. Finally, in the Appendix, we show, informally, how to find the rate function of the onedimensional LDP, by means of a contraction technique.

Some related work on large deviations for exponential functionals of BM can be found in Zani (2000, Chapter 5).

\section{A few facts about Bessel processes}

The Bessel process $\left(R_{t}^{(v)}, t \geqslant 0\right)$ of index $v$ is an $\mathbb{R}^{+}$-valued diffusion with infinitesimal generator $\mathscr{L}^{(v)}$ given by

$$
\left.\left.\mathscr{L}^{(v)} f(x)=\frac{1}{2} f^{\prime \prime}(x)+\frac{2 v+1}{2 x} f^{\prime}(x), \quad f \in \mathscr{C}_{b}^{2}(] 0,+\infty\right]\right) .
$$

Let $r>0$ and let $P_{r}^{(v)}$ denote the law of $\left(R_{t}^{(v)}, t \geqslant 0\right)$ starting from $R_{0}^{(v)}=r$ on $\mathscr{C}\left(\mathbb{R}_{+}, \mathbb{R}_{+}\right)$, the set of continuous functions from $\mathbb{R}_{+}$to $\mathbb{R}_{+}$. Let $\left(R_{t}, t \geqslant 0\right)$ be the canonical process on $\mathscr{C}\left(\mathbb{R}_{+}, \mathbb{R}_{+}\right)$, and $\mathscr{B}_{t}=\sigma\left\{R_{s}, s \leqslant t\right\}$ the canonical filtration. From Girsanov's theorem, for $v \geqslant 0$, the mutual absolute continuity relation holds:

$$
P_{r \mid \mathscr{R}_{t}}^{(v)}=\left(\frac{R_{t}}{r}\right)^{v} \exp \left\{\frac{-v^{2}}{2} \int_{0}^{t} \frac{\mathrm{d} s}{R_{s}^{2}}\right\} P_{r \mid \mathscr{R}_{t}}^{(0)} .
$$

If $v<0$, the Bessel process reaches 0 a.s.; and for $v<0$, we need to modify (12) as follows: 


$$
P_{r \mid \mathscr{R}_{t} \cap\left(t<T_{0}\right)}^{(v)}=\left(\frac{R_{t}}{r}\right)^{v} \exp \left\{\frac{-v^{2}}{2} \int_{0}^{t} \frac{\mathrm{d} s}{R_{s}^{2}}\right\} P_{r \mid \mathscr{R}_{t}}^{(0)},
$$

where $T_{0}=\inf \left\{u>0 ; R_{u}=0\right\}$.

Moreover, we shall need a slight extension of the family of Bessel processes, for dimension $\delta$ varying with time, $\delta(t)=2(1+v(t))$, and for $v$ a simple function given by

$$
\left.\left.v(t)=\sum_{i=1}^{n} v_{i}\right]_{1} t_{i}, t_{i+1}\right](t),
$$

and the $v_{i}$ are real numbers. The construction of such processes is done step by step on each time inverval: we still denote the corresponding law $P^{v(.)}$. It is easily seen that the absolute continuity property extends to this family; we leave the computation of the Radon-Nikodym densities to the reader.

\section{Large-deviation result}

Write

$$
S_{t}^{(\nu)}=\frac{1}{\log t} C_{t}^{(v)}
$$

We state an LDP for $\left(S_{t}^{(v)}, t \geqslant 0\right)$, and we show how this translates to $\left(A_{t}^{(v)}, t \rightarrow+\infty\right)$ via property (6).

Theorem 3.1. Let $\left(R_{t}^{(v)}, t \geqslant 0\right)$ be a Bessel process starting from $R_{0}^{(v)} \neq 0$ a.s., with dimension $d>2$. Set $v=d / 2-1$. Then $\left\{S_{t}^{(v)}\right\}$ satisfies an LDP with speed $(\log t)$ and good rate function

$$
\Lambda_{v}^{*}(x)=\frac{(2 v x-1)^{2}}{8 x}, \quad x \geqslant 0 .
$$

In particular, for any $s>0$,

$$
\frac{1}{\log t} P\left(S_{t}^{(v)}>\frac{1}{2 v}+s\right) \underset{t \rightarrow+\infty}{\longrightarrow}-\Lambda_{v}^{*}\left(\frac{1}{2 v}+s\right) .
$$

An immediate consequence is the following corollary:

Corollary 3.2. Let $\left(\beta_{u} ; u \geqslant 0\right)$ be a real-valued BM starting from $0, v>0$ and $A^{(v)}$ given by (5). Then, for any $a>0$,

$$
\frac{1}{v} \log P\left(\frac{1}{v} \log A_{v}^{(v)}<2 v-a\right) \underset{v \rightarrow+\infty}{\longrightarrow}-\frac{a^{2}}{8} .
$$

Proof of Corollary 3.2. This is immediate from Theorem 3.1 and relation (6). 
Proof of Theorem 3.1. With the notation introduced in Section 2 we obtain, for $\phi \leqslant v^{2} / 2$,

$$
\mathrm{E}_{r}^{(v)}\left(\mathrm{e}^{\phi C_{t}}\right)=\mathrm{E}_{r}^{\left(v_{\phi}\right)}\left(\frac{R_{t}}{r}\right)^{2 s(\phi)},
$$

where

$$
s(\phi)=\frac{1}{2}\left(v-v_{\phi}\right)
$$

and

$$
v_{\phi}=\sqrt{v^{2}-2 \phi}
$$

From the scaling property,

$$
\mathrm{E}_{r}^{\left(v_{\phi}\right)}\left(R_{t}\right)^{2 s(\phi)}=\mathrm{E}_{r / \sqrt{t}}^{\left(v_{\phi}\right)}\left(R_{1}\right)^{2 s(\phi)} t^{s(\phi)} .
$$

We compute the normalized cumulant generating function of $C_{t}$ :

$$
\Lambda_{\nu}^{t}(\phi):=\frac{1}{\log t} \log \mathrm{E}_{r}^{(v)}\left(\mathrm{e}^{\phi C_{t}}\right)=s(\phi)+\frac{1}{\log t} \log \left[\mathrm{E}_{r / \sqrt{t}}^{\left(v_{\phi}\right)}\left(R_{1}\right)^{2 s(\phi)}\right]-\frac{\log r^{2 s(\phi)}}{\log t} .
$$

Moreover,

$$
\mathrm{E}_{r / \sqrt{t}}^{\left(v_{\phi}\right)}\left(R_{1}\right)^{2 s(\phi)} \rightarrow \mathrm{E}_{0}^{\left(\nu_{(\phi)}\right)}\left(R_{1}\right)^{2 s(\phi)}
$$

as $t \rightarrow+\infty$, hence

$$
\Lambda_{t}^{v}(\phi) \rightarrow \Lambda_{v}(\phi) \equiv s(\phi) .
$$

Since $s$ is an essentially smooth function on its domain, we can apply the Gärtner-Ellis theorem (see Dembo and Zeitouni 1998). This yields the LDP, with rate function $\Lambda_{v}^{*}=s^{*}$, the Legendre dual of $s$.

Remark 3.3. Similar results hold for $\int_{a}^{t} \mathrm{~d} s / R_{s}^{2}$, where $a>0$. The only change is that $r$ should be replaced everywhere in the above proof by $R_{a} \neq 0$ a.s. Asymptotically, this makes no difference.

\section{Functional results}

In this section, we consider the functional version of Theorems 1.1 and 3.1. Let $\left(R_{t}^{(v)}, t \geqslant 0\right)$ be a $d$-dimensional Bessel process starting from $R_{0}^{(v)} \neq 0$ a.s. Denote by $\left\{Z_{n}^{(v)}\right\}$ the family of random functions defined on $[0,1]$ by

$$
u \rightarrow Z_{n}^{(v)}(u)=\frac{1}{n} \int_{1}^{\mathrm{e}^{n u}} \frac{\mathrm{d} s}{\left(R_{s}^{(v)}\right)^{2}} .
$$

Let $\mathscr{C}_{0}([0,1])$ be the set of continuous functions from $[0,1]$ to $\mathbb{R}$ such that $f(0)=0$, endowed with the supremum norm topology. We have the following theorem. 


\section{Theorem 4.1.}

(i) The family $\left\{Z_{n}^{(v)}\right\}_{n}$ converges a.s. on $\mathscr{C}_{0}([0,1])$ towards $u /(2 v)$.

(ii) The family $\left\{\sqrt{n}\left(Z_{n}^{(v)}-\mathrm{E}\left(Z_{n}^{(v)}\right)\right)\right\}$ converges in distribution in $\mathscr{C}_{0}([0,1])$ towards

$$
\left(\frac{1}{2 v} \gamma_{u}, 0 \leqslant u \leqslant 1\right)
$$

where $\left(\gamma_{u}, u \geqslant 0\right)$ is a Brownian motion.

For the proofs of Theorem 4.1 and 4.2, $v$ is fixed, and we make no further mention of this in the notation.

Proof. (i) We know that $Z_{n}(u) \rightarrow u / 2 v$ for any fixed $u \in[0,1]$. This is a family of increasing functions on a compact, converging pointwise (outside of a set of measure 0 ) to a continuous function. Hence from Dini's theorem, the convergence is uniform (a.s.).

(ii) From formula (9),

$$
\sqrt{n}\left(\frac{d-2}{2 n} \int_{1}^{\mathrm{e}^{n u}} \frac{\mathrm{d} s}{R_{s}^{2}}-\frac{u}{2}\right)+\frac{1}{\sqrt{n}} \int_{1}^{\mathrm{e}^{n u}} \frac{\mathrm{d} \beta_{s}}{R_{S}}=\frac{1}{\sqrt{n}} \log \left(\frac{R_{\mathrm{e}^{n u}}}{\mathrm{e}^{n u / 2}}\right) .
$$

Denote by $H_{n}(u)$ the above right-hand term. Then

$$
\sup _{u \leqslant A}\left|H_{n}(u)\right|=\frac{1}{\sqrt{n}} \sup _{1 \leqslant t \leqslant \mathrm{e}^{n A}}\left|\log \left(\frac{R_{t}}{\sqrt{t}}\right)\right|,
$$

which tends to 0 as $n \rightarrow \infty$, using on the one hand the law of the iterated logarithm, which takes care of the large values of $R_{t}$, and on the other hand the Dvoretsky-Erdős law (see, for example, Itô and McKean 1974) which takes care of the probability of $R_{t}$ being bounded away from 0 . Furthermore

$$
\frac{1}{\sqrt{n}} \int_{1}^{\mathrm{e}^{n u}} \frac{\mathrm{d} \beta_{s}}{R_{S}}=\gamma_{A_{u}^{(n)}}^{(n)}
$$

where

$$
A_{u}^{(n)}=\frac{1}{n} \int_{1}^{\mathrm{e}^{n u}} \frac{\mathrm{d} s}{R_{s}^{2}} .
$$

Now let us estimate, for any $\varepsilon>0$,

$$
p_{n}=P\left(\sup _{u \leqslant T}\left|\gamma_{A_{u}^{(n)}}^{(n)}-\gamma_{c u}^{(n)}\right| \geqslant \varepsilon\right),
$$

where $c=1 /(2 v)$. We can bound

$$
p_{n} \leqslant P\left(\sup _{u \leqslant T}\left|\gamma_{A_{u}^{(n)}}^{(n)}-\gamma_{c u}^{(n)}\right| \geqslant \varepsilon ; \sup _{u \leqslant T}\left|A_{u}^{(n)}-c u\right| \leqslant \delta\right)+P\left(\sup _{u \leqslant T}\left|A_{u}^{(n)}-c u\right| \geqslant \delta\right)
$$

From Bienaymé-Chebyshev, 


$$
p_{n} \leqslant \frac{1}{\varepsilon^{2}} \mathrm{E}\left[\sup _{u, \nu \leqslant 2 T}\left(\frac{\gamma_{u}^{(n)}-\gamma_{v}^{(n)}}{(v-u)^{1 / 3}}\right)^{2}\right] \delta^{2 / 3}+P\left(\sup _{u \leqslant T}\left|A_{u}^{(n)}-c u\right| \geqslant \delta\right) .
$$

Let us fix $\theta>0$. Since the first term on the right-hand side of (20) does not depend on $n$, we can fix $\delta$ small enough to have this term smaller than $\theta / 2$ (see, for example, Stroock and Varadhan 1979). Now, since $A_{u}^{n} \rightarrow u /(2 v)$, there exists $N$ large enough such that, for any $n \geqslant N$, the second right-hand side term of (20) is smaller than $\theta / 2$. This proves that $p_{n} \rightarrow 0$ as $n \rightarrow \infty$.

We also give a functional version of the previous LDP. The paths of $Z_{n}$ are increasing and continuous. Therefore they belong to $\mathscr{D}$, the space of cadlag functions on $[0,1]$. Let $\mathscr{C}$ be the set of functions $f[0,1] \rightarrow \mathbb{R}$ such that $f(0)=0$, with the topology of pointwise convergence. Let, for $\phi \in \mathscr{D}$

$$
\mathrm{I}(\phi)= \begin{cases}\int_{0}^{1} \Lambda_{v}^{*}\left(\dot{\phi}_{1}(s)\right) \mathrm{d} s+\frac{v^{2}}{2} \phi_{2}(1) & \text { if } \phi \text { is non-decreasing, } \phi(0)=0 \\ +\infty & \text { otherwise }\end{cases}
$$

where $\phi_{1}$ and $\phi_{2}$ are the absolutely continuous and singular components of $\phi$ (see de Acosta 1994; Lynch and Sethuraman 1987).

The singular part in the action functional comes from the fact that the underlying logLaplace transform is not finite everywhere; see formula (26).

Theorem 4.2. The family $\left\{Z_{n}^{(v)}\right\}$ satisfies an LDP in $\mathscr{C}$ with speed $n$ and good rate function I. which coincides with I on $\mathscr{D}$.

Proof. We proceed in three steps, detailed below. First, we state an LDP for a finitedimensional vector $\left(Z_{n}\left(u_{1}\right), Z_{n}\left(u_{2}\right), \ldots, Z_{n}\left(u_{p}\right)\right)$ for any $p$-tuple $\left(u_{1}, \ldots, u_{p}\right)$. Then a projective limit argument leads to a functional LDP in $\mathscr{X}$, endowed with the pointwise convergence topology. A small improvement on this argument gives the result of the theorem.

First step: Finite-dimensional result. Let $p \in \mathbb{N}$, and let $\mathbf{u}: 0<u_{1}<u_{2}<\ldots<u_{p} \leqslant 1$ be a subdivision of $[0,1]$. We set $t_{i}=\mathrm{e}^{n u_{i}}$, and we look for an LDP for the family $\left\{\left(Z_{n}\left(u_{1}\right), Z_{n}\left(u_{2}\right), \ldots, Z_{n}\left(u_{p}\right)\right)\right\}_{n \in \mathbb{N}}$.

Theorem 4.3. The family $\left\{\left(Z_{n}\left(u_{1}\right), Z_{n}\left(u_{2}\right), \ldots, Z_{n}\left(u_{p}\right)\right)\right\}_{n \in \mathbb{N}}$ satisfies an LDP with speed $n$ and good rate function defined by $I_{\mathbf{u}}\left(y_{1}, y_{2}, \ldots, y_{p}\right)= \begin{cases}\sum_{k=1}^{p}\left(u_{k}-u_{k-1}\right) \Lambda_{v}^{*}\left(\frac{y_{k}-y_{k-1}}{u_{k}-u_{k-1}}\right) & \text { if for every } i \in\{1, \ldots, p\}, y_{i}>y_{i-1}, \\ +\infty & \text { otherwise, }\end{cases}$

where $u_{0}=y_{0}=0$. 
Proof. Let $\left(\phi_{1}, \phi_{2}, \ldots, \phi_{p}\right) \in \mathbb{R}^{p}$. We compute the Laplace transform $\mathrm{E}_{r}^{v}\left(\exp \left\{\sum \phi_{k} \tilde{C}_{t_{k}}\right\}\right)$, where $t_{k}=\mathrm{e}^{n u_{k}}, k \geqslant 1, t_{0}=\mathrm{e}^{n u_{0}}=1$, and $\tilde{C}_{t_{k}=}=C_{t_{k}}-C_{t_{0}}$. From Girsanov,

$$
\mathrm{E}_{r}^{v}\left(\exp \left\{\sum_{k=1}^{p} \phi_{k} \tilde{C}_{t_{k}}\right\}\right)=\mathrm{E}_{r}^{v} \mathrm{E}_{R_{t_{0}}}^{0}\left(\exp \left\{\sum_{k=1}^{p-1} \phi_{k} \tilde{C}_{t_{k}}-\psi_{p} \tilde{C}_{t_{p}}\right\}\left(\frac{R_{t_{p}}}{R_{t_{0}}}\right)^{v}\right)
$$

where

$$
\psi_{p}=\frac{v^{2}}{2}-\phi_{p}
$$

Furthermore, the right-hand term of (22) can be written

$$
\mathrm{E}_{r}^{v}\left(\mathrm{E}_{R_{t_{0}}}^{0}\left(\exp \left\{\sum_{k=1}^{p-1} \phi_{k} \tilde{C}_{t_{k}}-\psi_{p} \tilde{C}_{t_{p+1}}\right\}\left(\frac{R_{t_{p}}}{R_{t_{0}}}\right)^{v}\left(\frac{R_{t_{p}}}{R_{t_{p-1}}}\right)^{-\sqrt{2 \psi_{p}}}\left(\frac{R_{t_{p}}}{R_{t_{p-1}}}\right)^{\sqrt{2 \psi_{p}}} \exp \left\{-\psi_{p}\left(\tilde{C}_{t_{p}}-\tilde{C}_{t_{p-1}}\right)\right\}\right)\right) .
$$

Proceeding in this manner, step by step, we can state that

$$
\mathrm{E}_{r}^{v}\left(\exp \left\{\sum_{k=1}^{p} \phi_{k} \tilde{C}_{t_{k}}\right\}\right)=\mathrm{E}_{r}^{v(.)}\left(R_{t_{0}}^{\alpha_{0}} R_{t_{1}}^{\alpha_{1}} R_{t_{2}}^{\alpha_{2}} \ldots R_{t_{p}}^{\alpha_{p}}\right)
$$

where $v(t)$ is a time-varying parameter for the changed Bessel process (see Section 2).

Let $v_{0}=v_{p+1}=0$ and for any $1 \leqslant k \leqslant p$,

$$
v_{k}=\sum_{i=k}^{p} \phi_{i}
$$

and let

$$
\mathscr{D}_{\Lambda}=\left\{\left(\phi_{1}, \phi_{2}, \ldots, \phi_{p}\right) ; \forall k \in\{1, \ldots, p\}, v_{k} \leqslant v^{2} / 2\right\} .
$$

Now if $g(v)=\sqrt{v^{2}-2 v}$, then

$$
v(t)=g\left(v_{k}\right) \quad \text { for } t \in\left(t_{k-1}, t_{k}\right], 1 \leqslant k \leqslant p, t_{-1}=0 .
$$

The exponents $\alpha_{k}$ are given by

$$
\alpha_{k}=g\left(v_{k+1}\right)-g\left(v_{k}\right) \quad v_{p+1}=0 .
$$

As in the previous section, we compute the normalized cumulant generating function. From the scaling property,

$$
\begin{aligned}
\Lambda_{n}^{\mathbf{u}}\left(\phi_{1}, \phi_{2}, \ldots, \phi_{p}\right) & =\frac{1}{n} \log \mathrm{E}_{r}^{v}\left(\exp \left\{\sum \phi_{i} C_{t_{i}}\right\}\right) \\
& =\frac{1}{n} \log \mathrm{E}_{r}^{v(.)}\left(R_{t_{0}}^{\alpha_{0}} R_{t_{1}}^{\alpha_{1}} R_{t_{2}}^{\alpha_{2}} \ldots R_{t_{p-1}}^{\alpha_{p-1}} \mathrm{E}_{R_{t_{p-1}}^{v\left(t_{p}\right)}}\left(R_{t_{p}-t_{p-1}}\right)^{\alpha_{p}}\right) \\
& =\frac{1}{n} \log \left(t_{p}-t_{p-1}\right)^{\alpha_{p} / 2}+\frac{1}{n} \log \mathrm{E}_{r}^{v(.)}\left(R_{t_{0}}^{\alpha_{0}} R_{t_{1}}^{\alpha_{1}} R_{t_{2}}^{\alpha_{2}} \ldots R_{t_{p-1}}^{\alpha_{p-1}} \mathrm{E}_{R_{t_{p-1}} / \sqrt{t_{p}-t_{p-1}}}^{v\left(t_{t}\right)}\left(R_{t_{0}}^{\alpha_{p}}\right)\right) .
\end{aligned}
$$

It is easily seen that 


$$
\frac{1}{n} \log \left(t_{p}-t_{p-1}\right)^{\alpha_{p} / 2}=\frac{1}{n} \log \left(\mathrm{e}^{n u_{p}}-\mathrm{e}^{n u_{p-1}}\right)^{\alpha_{p} / 2} \rightarrow \frac{\alpha_{p} u_{p}}{2} .
$$

Hence

$$
\Lambda_{n}^{\mathbf{u}} \rightarrow \Lambda_{\mathbf{u}},
$$

where

$$
\Lambda_{\mathbf{u}}\left(\phi_{1}, \phi_{2}, \ldots, \phi_{p}\right)= \begin{cases}\frac{1}{2} \sum_{k=1}^{p} \alpha_{k} u_{k} & \text { on } \mathscr{D}_{\Lambda} \\ +\infty & \text { otherwise. }\end{cases}
$$

We now determine the Fenchel-Legendre dual of $\Lambda_{\mathbf{u}}$ defined on $\mathbb{R}^{p}$ by

$$
\Lambda_{\mathbf{u}}^{*}(\mathbf{y})=\sup _{\phi \in \mathbb{R}^{p}}\left\{\mathbf{y} \cdot \phi-\Lambda_{\mathbf{u}}((\phi)\}\right.
$$

where $\mathbf{y}=\left(y_{1}, y_{2}, \ldots, y_{p}\right)$. Tedious but easy calculations lead to

$$
\Lambda_{\mathbf{u}}^{*}=I_{\mathbf{u}},
$$

where $I_{\mathbf{u}}$ is given by expression (21).

Second step: Projective limit. We now define a projective system so that we can transport the discrete result to a continuous one. Let $J$ denote the collection of all ordered finite subsets of $[0,1]$. A partial order by inclusion can be defined on $J$ as follows: for any $\mathbf{u}=\left\{0<u_{1} \leqslant u_{2} \leqslant \ldots \leqslant u_{p} \leqslant 1\right\}$ and $\mathbf{v}=\left\{0<v_{1} \leqslant v_{2} \leqslant \ldots \leqslant v_{q} \leqslant 1\right\}, \mathbf{u} \leqslant \mathbf{v}$ if and only if, $\forall i \leqslant p, u_{i}=v_{h(i)}$ for some applications $h: \mathbb{N} \rightarrow \mathbb{N}$. Set the natural projection $p_{\mathbf{u}, \mathbf{v}}$ :

$$
p_{\mathbf{u}, \mathbf{v}}: \mathbb{R}^{|\mathbf{u}|} \rightarrow \mathbb{R}^{|\mathbf{v}|}, \quad \mathbf{u} \leqslant \mathbf{v} .
$$

Denote $\mathscr{Y}_{\mathbf{u}}=\mathbb{R}^{|\mathbf{u}|}$ and let $\tilde{\mathscr{C}}$ be the projective limit of $\left(\mathscr{Y} \mathbf{u}, p_{\mathbf{u}, \mathbf{v}}\right)_{\mathbf{u} \leq \mathbf{v} \in \mathbf{J}}$. For $f \in \mathscr{C}$ and $\mathbf{u}=\left\{u_{1}, u_{2}, \ldots, u_{p}\right\} \in J$, let

$$
p_{\mathbf{u}}(f)=\left(f\left(u_{1}\right), f\left(u_{2}\right), \ldots, f\left(u_{p}\right)\right) .
$$

Actually $\tilde{\mathscr{C}}$ can be identified with $\mathscr{\mathscr { C }}$ as follows: for any $f \in \mathscr{X},\left(p_{\mathbf{u}}(f)\right)_{\mathbf{u} \in J} \in \tilde{\mathscr{X}}$ since $p_{\mathbf{u}}(f)=p_{\mathbf{u}, \mathbf{v}}\left(p_{\mathbf{v}}(f)\right)$ for any $\mathbf{u} \leqslant \mathbf{v}$. Conversely, for any $\left(x_{\mathbf{u}}\right)_{\mathbf{u} \in J}$ of $\tilde{\mathscr{C}}$, we can associate $f \in \mathscr{X}$ such that $p_{\mathbf{u}}(f)=x_{\mathbf{u}}$, i.e. $f\left(t_{i}\right)=x_{t_{i}}$. The projective topology on $\tilde{\mathscr{C}}$ coincides with the pointwise topology on $\mathscr{X}$. Note that the $\mathscr{Y} \mathbf{u}$ are Hausdorff spaces and that the $I_{\mathbf{u}}$ of Theorem 4.3 are good rate functions. Hence, we can apply the Dawson-Gärtner theorem (see Theorem 4.6.1 of Dembo and Zeitouni 1998): the family $\left\{Z_{n}\right\}$ satisfies an LDP with rate $n$ and good rate function

$$
I_{\mathscr{X}}(\phi)=\sup _{\mathbf{u} \in J} I_{\mathbf{u}}\left(p_{\mathbf{u}}(\phi)\right) .
$$

Third step: Conclusion. If $\phi$ is not increasing, $\mathrm{I}_{\mathscr{C}}(\phi)=+\infty$. If $\phi$ is increasing and cadlag, $\mathrm{I}_{\mathscr{C}}(\phi)=\mathrm{I}(\phi)$ as a consequence of Lynch and Sethuraman (1987) page 617. This ends the proof of Theorem 4.2. 


\section{Appendix}

The rate function of Theorem 3.1 can be found, informally, by the contraction principle (see Dembo and Zeitouni 1998). Let us consider for the sake of simplicity the case $\delta=d$ integer, $d>2$.

On the one hand, large deviations on $S_{t}$ can be carried to large deviations on as $(1 / t) \int_{0}^{t}\left|X_{s}\right|^{-2} \mathrm{~d} s$, via the classical transformation $X_{s}=\mathrm{e}^{-s / 2} R\left(\mathrm{e}^{s}\right)$, where $X_{s}$ is the $d$ dimensional stationary Ornstein-Uhlenbeck process with infinitesimal generator $L_{d}$ given by

$$
L_{d} \phi(x)=\frac{1}{2}(\Delta \phi(x)-\langle\nabla \phi(x), x\rangle) .
$$

On the other hand, let us consider the level 2 LDP for $X_{s}$, i.e. the LDP on the occupation measure $(1 / t) \int_{0}^{t} \delta_{X_{s}} \mathrm{~d} s$. From Donsker and Varadhan $(1975 \mathrm{a} ; 1975 \mathrm{~b} ; 1976 ; 1983)$, the rate function governing this LDP is

$$
I_{2}(\mu):=\frac{1}{2} \int_{\mathbb{R}^{d}}|\nabla f|^{2} \mathrm{~d} m_{0}=-\int_{\mathbb{R}^{d}} f(x) L_{d} f(x) \mathrm{d} m_{0}(x),
$$

where $m_{0}$ is the standard Gaussian measure, $\mu$ is a probability on $\mathbb{R}^{d}$ and $\mathrm{d} \mu / \mathrm{d} m_{0}=f^{2}$. The use of this result - and the classical transformation above - in large-deviation theory has been studied by Heck (1998) (see also March and Seppäläinen 1997).

Let us now consider a contraction. If $g \in \mathscr{C}_{b}\left(\mathbb{R}^{d}, \mathbb{R}\right)$, the mapping $\mu \mapsto \int g \mathrm{~d} \mu$ is continuous, and by the contraction principle, the LDP for $(1 / t) \int_{0}^{t} g\left(X_{s}\right) \mathrm{d} s$ has rate function

$$
I_{1}(a)=\inf \left\{I_{2}(\mu) ; g \mathrm{~d} \mu=a\right\} .
$$

If $g$ is a radial function, say $g(x)=\mathbf{g}\left(|x|^{2}\right)$, we may look for radial $f$, say $f(x)=\mathbf{f}\left(|x|^{2}\right)$. Let us denote by $\mathbf{m}_{0}$ the gamma distribution, the image of $m_{0}$ by the mapping $x \mapsto|x|^{2}$.

Since $\left|\nabla f(x)^{2}\right|^{2}=4|x|^{2} \mathbf{f}^{\prime}\left(|x|^{2}\right)^{2}$, the variational problem (28) becomes

$$
I_{1}(a)=\inf \left\{-\int_{0}^{\infty} \mathbf{f}(\rho) \mathbf{L}_{d} \mathbf{f}(\rho) \mathrm{d} \mathbf{m}_{0}(\rho) ; \int \mathbf{f}^{2}(\rho) \mathbf{g}(\rho) \mathrm{d} \mathbf{m}_{0}=a ; \int \mathbf{f}^{2}(\rho) \mathrm{d} \mathbf{m}_{0}(\rho)=1\right\},
$$

where $\mathbf{L}_{d}$ is the Laguerre infinitesimal generator, $\mathbf{L}_{d} \mathbf{f}(\rho)=2 \rho \mathbf{f}^{\prime \prime}(\rho)+(d-\rho) \mathbf{f}^{\prime}(\rho)$. This generator is the image of the radial part of $L_{d}$ by the mapping $r \rightarrow r^{2}$. From the Lagrange multipliers method, we find the differential equation satisfed by $\mathbf{f}$ :

$$
-\mathbf{L}_{d} \mathbf{f}(\rho)+\alpha \mathbf{f}(\rho) \mathbf{g}(\rho)+\beta \mathbf{f}(\rho)=0 .
$$

We wish to apply this scheme to obtain large deviations on $\int_{0}^{t}\left|X_{S}\right|^{-2} \mathrm{~d} s$. We try $\mathbf{g}(\rho)=\rho^{-1}$, which is far from continuous. In this case the partial differential equation (30) becomes

$$
-\mathbf{L}_{d} \mathbf{f}(\rho)+\alpha \rho^{-1} \mathbf{f}(\rho)+\beta \mathbf{f}(\rho)=0 .
$$

The only solution of (31) with the constraints as in (29) is

$$
\mathbf{f}_{a}(\rho)=C_{a} \rho^{s_{a}},
$$

with 


$$
s_{a}=\frac{1}{4 a}-\frac{v}{2} \quad \text { and } \quad C_{a}=2^{-s_{a}} \sqrt{\frac{\Gamma(v+1)}{\Gamma\left(2 s_{a}+v+1\right)}}, \quad v=\frac{d-2}{2} .
$$

For such an $\mathbf{f}_{a}$,

$$
I_{2}\left(f \mathrm{~d} m_{0}\right)=\frac{(2 v a-1)^{2}}{8 a},
$$

and we check

$$
I_{2}\left(f \mathrm{~d} m_{0}\right)=\Lambda_{v}^{*}(a) .
$$

\section{Acknowledgement}

The authors thank A. Rouault for many discussions and a careful reading of the paper.

\section{References}

de Acosta, A. (1994) Large deviations for vector-valued Lévy processes. Stochastic Process. Appl., 51, $75-115$.

Dembo, A. and Zeitouni, O. (1998) Large Deviations Techniques and Applications, 2nd edition. New York: Springer-Verlag.

Donsker, M. and Varadhan, S. (1975a) Asymptotic evaluation of certain Markov process expectations for large time. I. Comm. Pure Appl. Math., 28, 1-47.

Donsker, M. and Varadhan, S. (1975b) Asymptotic evaluation of certain Markov process expectations for large time. II. Comm. Pure Appl. Math., 28, 279-301.

Donsker, M. and Varadhan, S. (1976) Asymptotic evaluation of certain Markov process expectations for large time. III. Comm. Pure Appl. Math., 29, 389-461.

Donsker, M. and Varadhan, S. (1983) Asymptotic evaluation of certain Markov process expectations for large time. IV. Comm. Pure Appl. Math., 36, 183-212.

Geman, H. and Yor, M. (1993) Bessel processes, Asian option and perpetuities. Math. Finance, 3, $349-375$.

Heck, M. (1998) The principle of large deviations for the almost everywhere central limit theorem. Stochastic Process. Appl., 76, 61-75.

Hu, Y., Shi, Z. and Yor, M. (1999) Rates of convergence of diffusions with drifted Brownian potentials. Trans. Amer. Math. Soc., 351(10), 3915-3934.

Itô, K. and McKean, H.P. (1974) Diffusion Processes and Their Sample Paths. Berlin: Springer-Verlag.

Karlin, S. and Taylor, H. (1981) A Second Course in Stochastic Processes. New York: Academic Press.

Lynch, J. and Sethuraman, J. (1987) Large deviations for processes with independent increments. Ann. Probab., 15, 610-627.

March, P. and Seppäläinen, T. (1997) Large deviations from the almost everywhere central limit theorem. J. Theoret. Probab., 10, 935-965.

Revuz, D. and Yor, M. (1999) Continuous Martingales and Brownian Motion, 3rd edition. Berlin: Springer-Verlag.

Stroock, D. and Varadhan, S. (1979) Multidimensional Diffusion Processes. Berlin: Springer-Verlag. 
Warren, J. and Yor, M. (1997) Skew-products involving Bessel and Jacobi processes. Technical Report, Department of Mathematics, University of Bath.

Warren, J. and Yor, M. (1998) Perturbed Bessel processes. In J. Azéma, M. Émery, M. Ledoux and M. Yor (eds), Séminaire de Probabilités XXXII, Lecture Notes in Math. 1686, pp. 237-249. Berlin: Springer-Verlag.

Yor, M. (1992) On some exponential functionals of Brownian motion. Adv. Appl. Probab., 24, $509-531$.

Zani, M. (2000) Grandes déviations pour des fonctionnelles issues de la statistique des processus. Doctoral thesis, Université d'Orsay.

Received July 2000 